\title{
HOMOTOPY ANALYSIS NATURAL TRANSFORM METHOD FOR SOLVING FRACTIONAL PHYSICAL MODELS
}

\author{
S.Z. Rida ${ }^{1}$, A.A.A. Arafa ${ }^{2}$, A.S. Abedl-Rady ${ }^{1}$, H.R. Abdl-Rahim ${ }^{1}$ \\ ${ }^{1}$ Faculty of Science \\ South Valley University \\ Qena, EGYPT \\ ${ }^{2}$ Department of Mathematics and Computer Science \\ Faculty of Science \\ Port Said University \\ Port Said, EGYPT
}

\begin{abstract}
Homotopy analysis natural transform method (HANTM) is used to solve fractional physical models. This method is a combined form of the natural transform method and the homotopy analysis method. The fractional derivatives are described in the Caputo sense. The results reveal that the method is very effective, simple and can be applied to other fractional physical models.
\end{abstract}

AMS Subject Classification: homotopy analysis, natural transform method, fractional physical models

\section{Introduction}

Fractional Calculus has been used to model physical and engineering processes, which are found to be best described by fractional differential equations. It is worth nothing that the standard mathematical models of integer-order derivatives, including nonlinear models, do not work ade-quately in many cases. In

$\begin{array}{lr}\text { Received: } & 2017-01-07 \\ \text { Revised: } & 2017-07-15 \\ \text { Published: } & \text { November } 25,2017 \\ \text { Correspondence author }\end{array}$

$\S_{\text {Correspondence author }}$
(C) 2017 Academic Publications, Ltd. url: www.acadpubl.eu 
the recent years, fractional calculus has played a very important role in various fields an excellent literature of this can be found in (see [1-9]). There are several methods using for obtaining approximate analytical solutions of fractional physical models(see [10-18]). The purpose of this paper is to find approximate solutions for fractional nonlinear Benjamin-Bona-Mahony equation , fractional mod-ified Kawahara equation, and fractional Gardner equation via natural transform. The natural transform, initially was defined by Waqar et al. (See[19]) as the N - transform, which studied their properties and applications. Later, Belgacem et al. (see [20], [21]) defined its inverse and studied some additional fundamental properties of this integral transform and named it the natural transform. Applications of natural transform in the solution of differential and integral equations and for the distribution and Bohemians spaces can be found in (see [21-26]). Now, we mention the following basic definitions of natural transform and its properties as follows:

Definition 1. Over the set of functions

$$
A=\left\{f(t): \exists M, \tau_{1}, \tau_{2}>0,|f(t)|<M e^{|t| / \tau_{j}}, \text { if } t \in(-1)^{j} \times[0, \infty)\right\},
$$

the natural transform of $f(t)$ is

$$
N(f(t))=R(s, u)=\int_{0}^{\infty} f(u t) e^{-s t} d t, \quad u>0, s>0 .
$$

where $N[f(t)]$ is the natural transformation of the time function $f(t)$ and the variables $u$ and $s$ are the natural transform variables.

Theorem 1. We derives the relationship between natural and Laplace, Sumudu transform in successive theorems as follow(See[20]):

1. If $R(s, u)$ is the natural transform and $F(s)$ is the Laplace transform of function $f(t)$ in $A, G(u)$ is Sumudu transform then,

$$
N(f(t))=R(s, u)=\frac{1}{u} \int_{0}^{\infty} f(u t) e^{-s t} d t=\frac{1}{u} F\left(\frac{s}{u}\right)
$$

2. If $R(s, u)$ is the natural transform and $F(s)$ is the Laplace transform of function $f(t)$ in $A, G(u)$ is Sumudu transform then,

$$
N(f(t))=R(s, u)=\frac{1}{s} \int_{0}^{\infty} f\left(\frac{u t}{s}\right) e^{-t} d t=\frac{1}{s} G\left(\frac{u}{s}\right)
$$


3. If $f^{n}(t)$ is the nth derivative of function $f(t)$, then, its natural transform is given by:-

$$
N\left[f^{n}(t)\right]=R_{n}(s, u)=\frac{s^{n}}{u^{n}} R[s, u]-\sum_{k=0}^{n-1} \frac{s^{n-(k+1)}}{u^{n-k}} f^{(k)}(0+), n \geq 1
$$

4. If $F(s, u)$ and $G(s, u)$ are the natural transforms of respective functions $f(t)$ and $g(t)$ both defined in set $A$ then,

$$
N[f * g]=u F(s, u) G(s, u)
$$

where $f^{*} g$ is the convolution of two functions $f$ and $g$.

5. If $N[f(t)]$ is the natural transform of the function $f(t)$, then the natural transform of fractional derivative of order $\alpha$ is defined as:-

$$
N\left[f^{\alpha}(t)\right]=\frac{s^{\alpha}}{u^{\alpha}} R[s, u]-\sum_{k=0}^{n-1} \frac{s^{\alpha-(k+1)}}{u^{\alpha-k}} f^{(k)}(0+)
$$

6. Let the function $f(t)$ belongs to set $A$ be multiplied with weight functione $e^{ \pm t}$ then,

$$
N\left[f(t) e^{ \pm t}\right]=\frac{s}{(s+\mp u)} R[s, u]
$$

7. Let $f(t)$ the function belongs to set $A$, where a is non-zero constant then,

$$
N[f(a t)]=\frac{1}{a} R\left[\frac{s}{a}, u\right]
$$

8. If $w^{n}(t)$ is given by $w^{n}(t)=\int_{0}^{t} \ldots \int_{0}^{t} f(t)(d t)^{n}$, then, the natural transform of $w^{n}(t)$ is given by

$$
N\left[w^{n}(t)\right]=\frac{u^{n}}{s^{n}} R(s, u)
$$

9. The natural transform of T-periodic function $f(t) \in A$ such thatf $(t+$ $n T)=f(t), n=0,1,2, \ldots$ is given by:

$$
N[f(t)]=R(s, u)=\left[1-e^{-\left(\frac{s T}{u}\right)}\right]^{-1} \frac{1}{u} \int_{0}^{t} e^{-\left(\frac{s T}{u}\right)} f(t) d t
$$

10. The function $f(t)$ in set $A$ is multiplied with shift function $t^{n}$, then,

$$
N\left[t^{n} f(t)\right]=\left(\frac{u^{n}}{s^{n}}\right) \frac{d^{n}}{d u^{n}} u^{n} R(s, u)
$$




\section{Analysis of the Method}

To illustrate the basic idea of homotopy analysis natural transform method, we consider an equation, where $\mathrm{F}$ represents a general nonlinear ordinary or partial differential operator including both linear and nonlinear terms. The linear terms are decomposed into $\mathrm{L}+\mathrm{R}$, where $\mathrm{L}$ is the highest order linear operator and $\mathrm{R}$ is the remaining of the linear operator. Thus, the equation can be written as:

$$
L U+R U+F U=g(x)
$$

where $F[U]]$ indicates the nonlinear terms.

By applying the natural transform on both sides of equation (2.1), we get

$$
N[L U]+N[R U]+N[F U]=N[g(x)]
$$

Using the differentiation property of the natural transform, we have

$$
\frac{s^{n}}{u^{n}} N[U]-\sum_{k=0}^{n-1} \frac{s^{n-(k+1)}}{u^{n-k}} U^{(k)}(0+)+N[R U]+N[F U]=N[g(x)]
$$

After simplifying, we have

$$
N[U]-\frac{u^{n}}{s^{n}} \sum_{k=0}^{n-1} \frac{s^{n-(k+1)}}{u^{n-k}} U^{(k)}(0+)+\frac{u^{n}}{s^{n}}[N[R U]+N[F U]-N[g(x)]]=0
$$

We define the nonlinear operator

$$
\begin{gathered}
F[\phi(x, t ; q)]=N[\phi(x, t ; q)]-\frac{u^{n}}{s^{n}} \sum_{k=0}^{n-1} \frac{s^{n-(k+1)}}{u^{n-k}} \phi^{(k)}(x, t ; q)(0+) \\
+\frac{u^{n}}{s^{n}}[N[R \phi(x, t ; q)]+N[F \phi(x, t ; q)]-N[g(x)]],
\end{gathered}
$$

where $q \in[0,1]$ and $\phi(x, t ; q)$ is a real function of $\mathrm{x}, \mathrm{t}$ and $\mathrm{q}$. We construct a homotopy as follows:

$$
(1-q) N\left[\phi(x, t ; q)-U_{0}(x, t)\right]=q \hbar H(x, t) F[U(x, t)]
$$

where $q \in[0,1]$ is the embedding parameter, $\hbar \neq 0$ is an auxiliary parameter, $N$ is denotes the natural transform, $U_{0}(x, t)$ is an initial guess of $U(x, t)$ and 
$\phi(x, t ; q)$ is an unknown function of $x, t$ and $H(x, t)$ is a nonzero auxiliary function.

Obviously, when the embedding parameter $q=0$ and $q=1$, it holds

$$
\phi(x, t ; 0)=U_{0}(x, t), \phi(x, t ; 1)=U(x, t)
$$

respectively. Thus as $q$ increases from 0 to 1 , the solution $\phi(x, t ; q)$ varies from the initial guess $U_{0}(x, t)$ to the solution $U(x, t)$. Expand $\phi(x, t ; q)$ in Taylor series with respect to $q$, we have:

$$
\phi(x, t ; q)=U_{0}(x, t)+\sum_{m=1}^{\infty} U_{m}(x, t) q^{m},
$$

where

$$
U_{m}(x, t)=\left.\frac{1}{m !} \frac{\partial^{m} \phi(x, t ; q}{\partial q^{m}}\right|_{q=0}
$$

If the auxiliary linear operator, the initial guess, the auxiliary parameter $\hbar$, and the auxiliary function are properly chosen, the series (2.8) converges at $\mathrm{q}=1$, then we have:

$$
U(x, t)=U_{0}(x, t)+\sum_{m=1}^{\infty} U_{m}(x, t)
$$

Defined the vectors

$$
U_{m}^{\rightarrow}(x, t)=\left\{U_{1}(x, t), U_{2}(x, t), U_{3}(x, t), \ldots, U_{m}(x, t)\right\} .
$$

Differentiating Eq. (2.7) times with respect to $\mathrm{q}$ and then divided by $\mathrm{m}$ ! and finally setting $\mathrm{q}=0$, we get the following $\mathrm{mth}$-order deformation equation

$$
N\left[U_{m}(x, t)-\chi_{m} U_{m-1}(x, t)\right]=\hbar R_{m}\left(U_{m-1}^{\rightarrow}\right),
$$

where

$$
R_{m}\left(U_{m-1}^{\rightarrow}(x, t)\right)=\left.\frac{1}{m !} \frac{\partial^{m-1} F[\phi(x, t ; q)]}{\partial q^{m-1}}\right|_{q=0}
$$

and

$$
\left\{\begin{array}{l}
0, m \leq 1 \\
1, m<1
\end{array}\right.
$$

Applying the inverse natural transform, we have

$$
U_{m}(x, t)=\chi_{m} U_{m-1}(x, t)+\hbar N^{-1}\left[R_{m}\left(U_{m-1}^{\rightarrow}\right)\right], m=0,1,2, \ldots
$$




\section{Applications}

\subsection{Benjamin-Bona-Mahony Equation}

Benjamin-Bona-Mahony equation was first proposed in 1972 by Benjamin et al.(See[28]). This equation is an alternative to the Korteweg-de Vries (KdV) equation, and describes the unidirectional propagation of small-amplitude long waves on the surface of water in channel. The BBM equation is not only convenient for shallow water waves but also for hydro magnetic waves, acoustic waves, and therefore it has more advantages compared with the KdV equation. Consider fractional nonlinear Benjamin-Bona-Mahony equation (see [29]):

$$
D_{t}^{\alpha}-U_{x x t}+U U_{x}+U_{x}=0,0<\alpha \leq 1, t>0
$$

subject to initial condition:

$$
U(x, 0)=\operatorname{sech}^{2}\left(\frac{x}{4}\right)
$$

According to the HANTM, we take the initial guess as:

$$
U(x, 0)=\operatorname{sech}^{2}\left(\frac{x}{4}\right)
$$

By applying natural transform on both sides of Eq.(3.1) subject to initial condition, we have

$$
N\left[D_{t}^{\alpha}\right]-N\left[U_{x x t}\right]+N\left[U U_{x}\right]+N\left[U_{x}\right]=0
$$

Using the differentiation property of the natural transform, we have

$$
\frac{s^{\alpha}}{u^{\alpha}} N[U]-\sum_{k=0}^{n-1} \frac{s^{\alpha-(k+1)}}{u^{\alpha-k}} U^{(k)}(0+)-N\left[U_{x x t}\right]+N\left[U U_{x}\right]+N\left[U_{x}\right]=0
$$

After simplifying, we have

$$
N[U]-\frac{1}{s} U^{(k)}(0+)-\frac{u^{\alpha}}{s^{\alpha}} N\left[U_{x x t}\right]+\frac{u^{\alpha}}{s^{\alpha}} N\left[U U_{x}\right]+\frac{u^{\alpha}}{s^{\alpha}} N\left[U_{x}\right]=0
$$

We define the nonlinear operator

$$
\begin{aligned}
F[\phi(x, t ; q)]= & N[\phi(x, t ; q)]-\frac{1}{s} U^{(k)} \phi(x, t ; q)(0+)-\frac{u^{\alpha}}{s^{\alpha}} N\left[\phi_{x x t}(x, t ; q)\right]+ \\
& \frac{u^{\alpha}}{s^{\alpha}} N\left[\phi(x, t ; q) \phi_{x}(x, t ; q)\right]+\frac{u^{\alpha}}{s^{\alpha}} N\left[\phi_{x}(x, t ; q)\right]=0
\end{aligned}
$$


Thus,

$$
\begin{aligned}
\Re_{m}\left[U_{m-1}^{\rightarrow}\right]= & N\left[U_{m-1}\right]-\left(1-\chi_{m}\right)\left(\frac{1}{s}\right) \operatorname{sech}^{2}\left(\frac{x}{4}\right)-\frac{u^{\alpha}}{s^{\alpha}} N\left[\left(U_{m-1}\right)_{x x t}\right] \\
& +\frac{u^{\alpha}}{s^{\alpha}} N\left[\left(\sum_{r=0}^{m-1} U_{r}\left(U_{m-r-1}\right)_{x}\right)\right]+\frac{u^{\alpha}}{s^{\alpha}} N\left[\left(U_{m-1}\right)_{x}\right]
\end{aligned}
$$

The $m^{\text {th }}$ order deformation equation is given by:

$$
\left.N\left[U_{m}(x, t)-\chi_{m} U_{m-1}(x, t)\right]=\hbar \Re\left(U_{m-1}^{\rightarrow}\right)\right]
$$

Applying the inverse natural transform, we have

$$
U_{m}(x, t)=\chi_{m} U_{m-1}(x, t)+\hbar N^{-1}\left[\Re\left(U_{m-1}^{\rightarrow}\right)\right]
$$

By solving above equation for $\mathrm{m}=1,2, \ldots$ we get:

$$
\begin{gathered}
U_{1}=-\frac{1}{2} \frac{t^{\alpha}}{\Gamma(\alpha+1)} \hbar \operatorname{sech}^{2}\left(\frac{x}{4}\right) \tanh \left(\frac{x}{4}\right)\left[1+\operatorname{sech}^{2}\left(\frac{x}{4}\right)\right] \\
U_{2}=\hbar(\hbar+1) \frac{t^{\alpha}}{\Gamma(\alpha+1)}\left[-\frac{1}{2} \operatorname{sech}^{4}\left(\frac{x}{4}\right) \tanh \left(\frac{x}{4}\right)-\frac{1}{2} \operatorname{sech}^{2}\left(\frac{x}{4}\right) \tanh \left(\frac{x}{4}\right)\right] \\
+\hbar^{2}\left(\frac{t^{2} \alpha}{\Gamma(2 \alpha+1)}\right)\left[\frac{3}{4} \operatorname{sech}^{6}\left(\frac{x}{4}\right) \tanh ^{2}\left(\frac{x}{4}\right)+\operatorname{sech}^{4}\left(\frac{x}{4}\right) \tanh ^{2}\left(\frac{x}{4}\right)\right. \\
\left.+\frac{1}{4} \operatorname{sech}^{2}\left(\frac{x}{4}\right) \tanh ^{2}\left(\frac{x}{4}\right)-\frac{1}{8} \operatorname{sech}^{2}\left(\frac{x}{4}\right)\right] \\
\left.-\frac{1}{4} \operatorname{sech}^{6}\left(\frac{x}{4}\right)-\frac{1}{8} \operatorname{sech}^{8}\left(\frac{x}{4}\right)\right]
\end{gathered}
$$

The other components of the HANTM can be determined in a similar way. Finally, the approximate solution of Eq. (3.1) in a series form is given by taking $\hbar=-1$ as follows

$$
U(x, t)=\sum_{m=0}^{\infty} U_{m}(x, t)=U_{0}+U_{1}+U_{2}+U_{3}+\ldots
$$




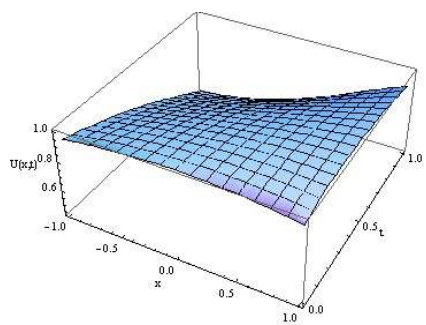

(a)

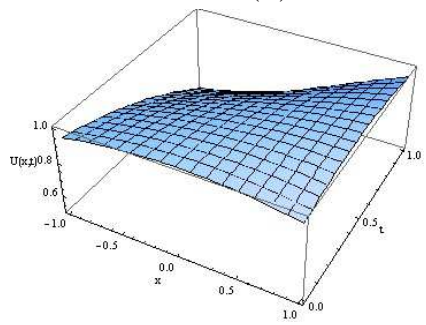

(c)

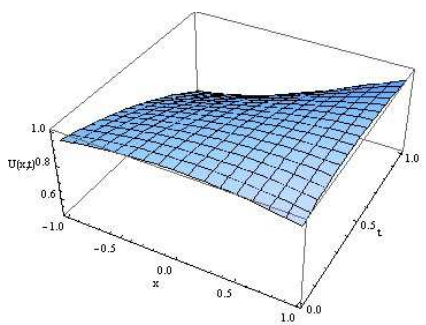

(b)

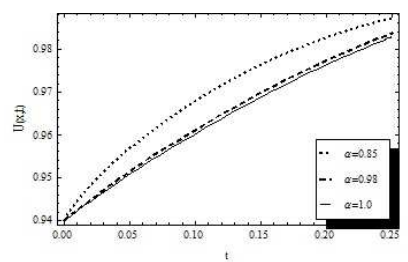

(d)

Figure 1: The approximate solution of second order of $U(x, t)$ of application 1 when (a) $\alpha=0.85$, (b) $\alpha=0.98$, (c) $\alpha=1$ which is the exact solution, and plot2D of second order of $U(x, t)$ versus $\mathrm{t}$ at $\mathrm{x}=1$ for different values of $\alpha$ and comparison the results with the exact solution as shown in $(d)$.

\subsection{Modified Kawahara Equation}

A variety of physical phenomena like, magneto acoustic waves in plasma(see [30]), shallow water waves with surface tension (see [31] ) and capillary gravity water waves (see [32]), are described by Kawahara Equation, modified Kawahara Equation, and KdV-Kawahara Equation. Consider fractional modified Kawahara equation(See[33]):

$$
D_{t}^{\alpha} U(x, t)+U_{x x}(x, t)+U^{2}(x, t) U_{x}(x, t)-U_{x x x x x}(x, t)=0,0<\alpha \leq 1, t>0
$$

subject to initial condition:

$$
U(x, 0)=D_{\operatorname{sech}^{2}}(k x)
$$

According to the HANTM, we get:

$$
U(x, 0)=D_{\operatorname{sech}}^{2}(k x),
$$




$$
\begin{aligned}
& U_{1}=\hbar \frac{t^{\alpha}}{\Gamma(\alpha+1)}\left[272 D k^{5} \operatorname{sech}^{6}(k x) \tanh (k x)-416 k^{5} \operatorname{sech}^{4}(k x) \tanh ^{3}(k x)\right. \\
& -2 D k^{2} \operatorname{sech}^{4}(k x)+4 D k^{2} \operatorname{sech}^{2}(k x) \tanh ^{2}(k x)+32 D k^{5} \operatorname{sech}^{2}(k x) \\
& \left.\tanh ^{5}(k x)-2 D^{3} k \operatorname{sech}^{6}(k x) \tanh (k x)\right] \\
& U_{2}=\hbar(\hbar+1) \frac{t^{\alpha}}{\Gamma(\alpha+1)}\left[272 D k^{5} \operatorname{sech}^{6}(k x) \tanh (k x)-416 k^{5} \operatorname{sech}^{4}(k x)\right. \\
& \tanh ^{3}(k x)-2 D k^{2} \operatorname{sech}^{4}(k x)+4 D k^{2} \operatorname{sech}^{2}(k x) \tanh ^{2}(k x)+32 D k^{5} \\
& \left.\operatorname{sech}^{2}(k x) \tanh ^{5}(k x)-2 D^{3} k \operatorname{sech}^{6}(k x) \tanh (k x)\right]+\hbar^{2} \frac{t^{2 \alpha}}{\Gamma(\alpha+1)}\left[8 D k^{3}\right. \\
& \operatorname{sech}^{2}(k x)\left(-992 k^{4} \operatorname{sech}^{6}(2 k x) \tanh (k x)+2 \operatorname{sech}^{4}(k x)\left(k+1536 k^{4} \operatorname{sech}^{3}(k x)\right)\right. \\
& +\operatorname{sech}^{2}(k x) \tanh (k x)\left(2 D^{2} \operatorname{sech}^{4}(k x)-11 k \tanh (k x)-960 k^{4} \tanh ^{4}(k x)\right) \\
& +\operatorname{tant}^{3}(k x)\left(-D^{2} \operatorname{sech}^{4}(k x)+2 k \tanh (k x)+16 k^{4} \tanh ^{4}(k x)\right)+\frac{1}{4} \frac{t^{2 \alpha}}{\Gamma(\alpha+1)} \\
& D^{3} k^{2} h^{2} \operatorname{sech}^{6}(k x)\left[8 D^{2}(-2+\cosh (2 k x)) \operatorname{sech}^{6}(k x)-k \operatorname{sech}^{6}(k x)\left(-9664 k^{3}\right.\right. \\
& +9528 k^{3} \cosh (2 k x)-960 k^{3} \cosh (4 k x)+8 k^{3} \cosh (6 k x)-19 \sinh (2 k x)-8 \sinh (4 k x) \\
& +\sinh (6 k x))-4 k \operatorname{sech}^{5}(k x)\left(-8 \cosh (k x)+2 \sinh (k x)\left(98 k^{3}-448 k^{3} \cosh (2 k x)\right.\right. \\
& \left.+8 k^{3} \cosh (4 k x)+\sinh (4 k x) \tanh (k x)+32 D^{2} \operatorname{sech}^{4}(k x) \tanh ^{2}(k x)\right] \\
& -\frac{1}{4}\left(\frac{t^{2 \alpha}}{\Gamma(2 \alpha+1)}\right)\left(D k ^ { 6 } \hbar ^ { 2 } \operatorname { s e c h } ^ { 8 } ( k x ) \left[8 D^{2}(-1208+1191 \cosh (2 k x)-120 \cosh (4 k x)\right.\right. \\
& +\cosh (6 k x)) \operatorname{sech}^{4}(k x)+k \operatorname{sech}^{4}(k x)\left(62896992 k^{3}-77904912 k^{3} \cosh (2 k x)\right. \\
& +17627904 k^{3} \cosh (4 k x)-1221096 k^{3} \cosh (6 k x)+244 \sinh (8 k x)-3555 \sinh (6 k x) \\
& \left.\left.+16288 k^{3} \cosh (4 k x)-8 k^{3} \cosh (10 k x)+18606 \sinh (2 k x)+3480 \sinh (4 k x)\right)\right)
\end{aligned}
$$

The other components of the HANTM can be determined in a similar way. Finally, the approximate solution of Eq. (3.14) in a series form is given by taking $\hbar=-1$ as follows

$$
U(x, t)=\sum_{m=0}^{\infty} U_{m}(x, t)=U_{0}+U_{1}+U_{2}+U_{3}+\ldots
$$




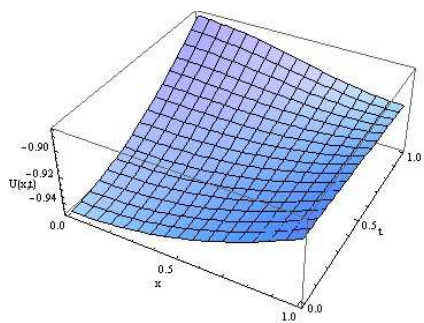

(a)

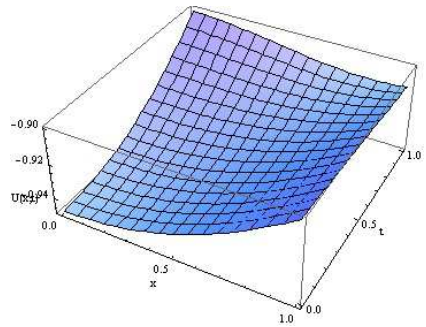

(c)

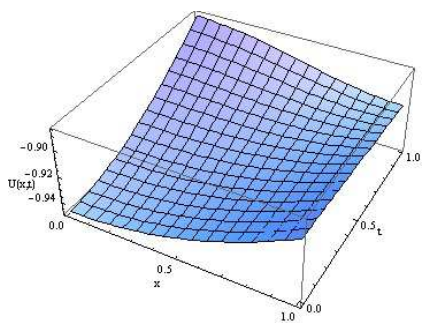

(b)

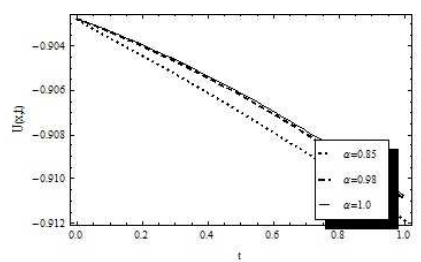

(d)

Figure 2: The approximate solution of second order of $U(x, t)$ of application 2 when (a) $\alpha=0.85$, (b) $\alpha=0.98$, (c) $\alpha=1$ which is the exact solution, and plot2D of second order of $U(x, t)$ versus $\mathrm{t}$ at $\mathrm{x}=1$, $D=\frac{-3}{\sqrt{10}}$, and $k=\frac{1}{2 \sqrt{5}}$ for different values of $\alpha$ and comparison the results with the exact solution as shown in $(d)$.

\subsection{Gardner Equation}

The Gardner equation is a useful model for the description of internal solitary waves in shallow water. Consider fractional Gardner equation(See[34]) :

$$
D_{t}^{\alpha}-U_{x x x}-6 U^{2} U_{x}-6 U=0,0<\alpha \leq 1, t>0
$$

subject to initial condition:

$$
U(x, 0)=-\frac{1}{2}\left(1-\tanh \left(-\frac{x}{2}\right)\right)
$$

As the previous applications, by applying the HANTM we have:

$$
\begin{gathered}
U(x, 0)=-\frac{1}{2}\left(1-\tanh \left(-\frac{x}{2}\right)\right) \\
U_{1}=\frac{1}{8} \hbar \frac{t^{\alpha}}{\Gamma(\alpha+1)}\left[-3 \operatorname{sech}^{2}\left(\frac{x}{2}\right)+\operatorname{sech}^{4}\left(\frac{x}{2}\right)+8\left(3+6 \operatorname{cosech}^{3}(x)\right.\right.
\end{gathered}
$$




$$
\begin{gathered}
\left.\sinh ^{4}\left(\frac{x}{2}\right)-10 \operatorname{cosech}^{4}(x) \sinh ^{6}\left(\frac{x}{2}\right)-3 \tanh \left(\frac{x}{2}\right)\right] \\
U_{2}=(1+\hbar)\left[\frac { 1 } { 8 } \hbar \frac { t ^ { \alpha } } { \Gamma ( \alpha + 1 ) } \left[-3 \operatorname{sech}^{2}\left(\frac{x}{2}\right)+\operatorname{sech}^{4}\left(\frac{x}{2}\right)+8\left(3+6 \operatorname{cosech}^{3}(x) \quad(3.24)\right.\right.\right. \\
\left.\left.\sinh ^{4}\left(\frac{x}{2}\right)-10 \operatorname{cosech}^{4}(x) \sinh ^{6}\left(\frac{x}{2}\right)-3 \tanh \left(\frac{x}{2}\right)\right]\right] \\
+\frac{1}{128} \hbar^{2} \frac{t^{2 \alpha}}{\Gamma(2 \alpha+1)} \operatorname{sech}^{7}\left(\frac{x}{2}\right)\left[\cos \left(\frac{x}{2}\right) \sinh \left(\frac{x}{2}\right)\right] \\
{[-2+1775 \cosh (x)+110 \cosh (2 x)+205 \cosh (3 x)-749 \sinh (x)+344 \sinh (2 x)} \\
-133 \sinh (3 x)]
\end{gathered}
$$

The other components of the HANTM can be determined in a similar way. Finally, the approximate solution of Eq. (26) in a series form is given by taking $\hbar=-1$ as follows:

$$
U(x, t)=\sum_{m=0}^{\infty} U_{m}(x, t)=U_{0}+U_{1}+U_{2}+U_{3}+\ldots
$$

\section{Conclusion}

In this paper, we have successfully used homotopy analysis natural transform method for solving fractional nonlinear Benjamin-Bona-Mahoney equation, fractional modified Kawahara equation, and fractional Gardner equation. Our results confirm that the method is very powerful and efficient technique for handling solutions of a class of fractional physical models.

\section{References}

[1] K. B. Oldham and J. Spanier, The Fractional Calculus, Academic Press, New York, NY, USA, 1974.

[2] I. Podlubny, Fractional Differential Equations, Academic Press, New York, NY, USA, 1999

[3] A.A. Kilbas, H.M. Srivastava, J.J. Trujillo, Theory and Applications of Fractional Differential Equations, Elsevier, Amsterdam, The Netherlands, 2006.

[4] M. Caputo, Linear models of dissipation whose Q is almost frequency independent, part II, Geophysical Journal International, 13 (1967), 529-539. 


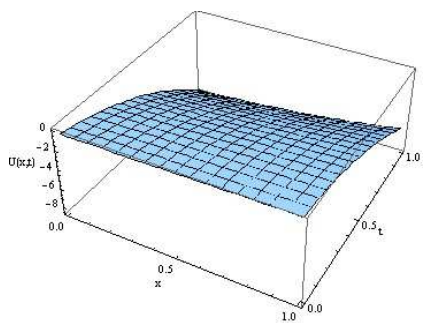

(a)

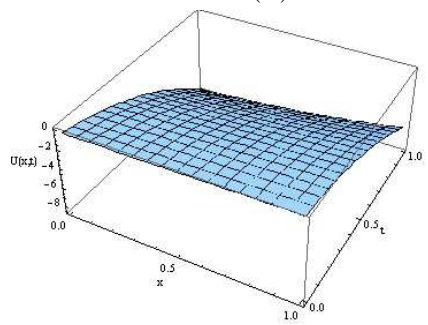

(c)

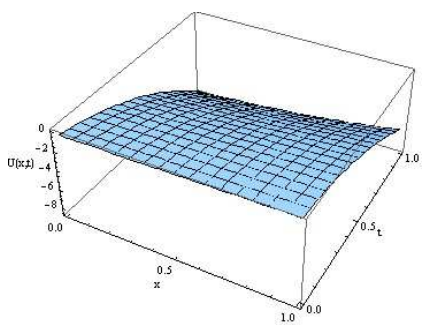

(b)

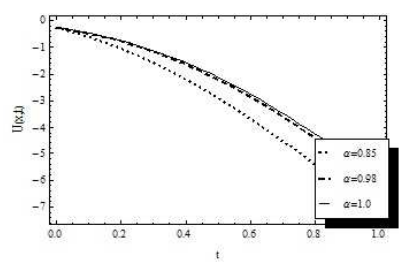

(d)

Figure 3: The approximate solution of second order of $U(x, t)$ of application 3 when (a) $\alpha=0.85$, (b) $\alpha=0.98$, (c) $\alpha=1$ which is the exact solution, and plot2D of second order of $U(x, t)$ versus $\mathrm{t}$ at $\mathrm{x}=1$ for different values of $\alpha$ and comparison the results with the exact solution as shown in $(d)$.

[5] K.S. Miller, B. Ross, An Introduction to the Fractional Calculus and Fractional Differential Equations, Wiley, New York, NY, USA, 1993.

[6] S.G. Samko, A.A. Kilbas, O.I. Marichev, Fractional Integrals and De-rivatives: Theory and Applications, Gordon and Breach, Yverdon, Swit-zerland, 1993.

[7] G. M. Zaslavsky, Hamiltonian Chaos and Fractional Dynamics, Oxford Uni-versity Press, 2005.

[8] A. Yildirim, An algorithm for solving the fractional nonlinear Schrodinger equation by means of the homotopy perturbation method, International Journal of Nonlinear Sciences and Numerical Simulation, 10 (2009), 445-450.

[9] H. Bulut, H.M. Baskonus, F.B. M.Belgacem, The analytical solution of some fractional ordinary differential equations by the Sumudu transform method, Abstract and Applied Analysis, 2013, 1-6.

[10] A.A.M. Arafa, S.Z. Rida, Numerical modeling for some generalized coupled nonlinear evolution equations, Mathematical and Computer Modelling, 56 (2012), 268-277.

[11] S.Z. Rida, A.M.A. El-Sayed, A.A.M. Arafa, Effect of bacterial memory dependent growth by using fractional derivatives reaction-diffusion chemotactic model, J. Statistical Physics, 140 (2010), 797-811. 
[12] A.M.A. El-Sayed, S. Z. Rida, A.A.M. Arafa, On the Solutions of the generalized reactiondiffusion model for bacteria growth, Acta Appl. Math., 110 (2010), 1501-1511.

[13] A.A.M. Arafa, S.Z. Rida, M. Khalil, The effect of anti-viral drug treatment of human immunodeficiency virus type 1 (HIV 1) described by a fractional order mode, Applied Mathematical Modeling, 37 (2013), 2189-2196.

[14] A.A.M. Arafa, S.Z. Rida, H. Mohamed, Approximate analytical solutions of Schnakenberg systems by homotopy analysis method, Applied Mathematical Modelling, 36 (2012), 4789-4796.

[15] A.A.M. Arafa, S.Z. Rida, A.A. Mohammadein, H.M. Ali, Solving nonlinear fractional differential equation by generalized Mittag-Leffler function method, Commun. Theor. Physics, 59 (2013) 661-663.

[16] A.A.M. Arafa, S.Z. Rida, H. Mohamed, Homotopy analysis method for solving biological population model, Commun. Theor. Phys., 56 (2011), 797-800.

[17] S.Z. Rida, A.A.M. Arafa, New method for solving linear fractional differential equations, International Journal of Differential Equations, 2011, 1-8.

[18] A.A.M. Arafa, S.Z. Rida, M. Khalil, A fractional-order model of HIV infection: Numerical solution and comparisons with data of patients, International Journal of Biomathematics, 7 (2014), 1-11.

[19] Deshna Loonker and P.K. Banerji, Natural transform for distribution and Boehmian spaces, Math. Engg. Sci. Aerospace, 4 (2013), 69-76.

[20] Deshna Loonker and P.K. Banerji, Natural transform and solution of inte-gral equations for distribution spaces, Amer. J. Math. Sci. (2013).

[21] Deshna Loonker and P. K. Banerji, Applications of natural transform to differential equations, J. Indian Acad. Math., 35 (2013), 151-158.

[22] G.M. Mittag-Leffer, Sur la nouvelle function, C. R. Acad. Sci., Paris, 137 (1903), 554558.

[23] R. Silambarasan and F. B. M. Belgacem, Theory of natural transform, Mathematics in Engineering, Science and Aerospace (MESA), 3 (2012), 99-124.

[24] H.M. Baskonus, H. Bulut, and Y. Pandir, The natural transform decomposition method for linear, Mathwmatics in engineering, science and aerospace, Mathematics in Engineering, Science and Aerospace (MESA), 5 (2014), 111-126.

[25] P. Mosconi, G. Mussardo, and V. Rida, Boundary quantum field theories with infinite resonance states, Nuc. Phys. B, 631 (2002).

[26] T. B. Benjamin, J. L. Bona, and, J.J. Mahony, Model equations for long waves in nonlinear dispersive system philos, Trans. Soc., London S.R., 272 (1972), 47-78.

[27] B. Hasan, H. B. Mehmet, T. Seyma, and A. Tojga, A comparison between HPM and ADM for the nonlinear Benjamin-Bona-Mahony equation, International Journal of Basic and Applied Science, 11 (2011), 117-127.

[28] T. kawahara, Osciallatory, solitary waves in dispersive media, Journal of Physical Society in Japan, 33 (1972), 260-264.

[29] T. Bridges and G. Derks, Linear instability of solitary wave solutions of the Kawahara quation and its en-eralizations, Society for Industrial and Applied Mathematics: Journal on Mathematical Analysis, 33 (2002), 1356-1378. 
[30] D.J. Scheurle, Existence of Perturbed solitary wave solutions to a model equation for water waves, Physica D, 32 (1988), 253-268.

[31] K.T. Alligood, T.D. Sauer, J.A. Yorke, Chaos: An Introduction to Dynamical Systems, Springer, 1996.

[32] A.M. Wazwaz, New Solitary Wave Solutions to the Modified Kawahara Equation, Physics Le tetra, 360 (2007), 588-592.

[33] R.M. May, Simple mathematical models with very complicated dynamics, Nature, 261 (1976), 459-467.

[34] A.M. Wazwaz, New solitons and kink solutions for the Gardner equation, Comm. Nonlin. Sci. Numer. Simul., 12 (2007), 1395-404. 